\title{
Resistência a $\beta$-lactâmicos em Acinetobacter spp isolados de efluente hospitalar no sul do Brasil
}

\author{
Resistance to $\beta$-lactams among Acinetobacter spp isolated \\ from hospital sewage in southern Brazil
}

\author{
Carolina de Souza Gusatti ${ }^{1}$, Alessandra Einsfeld Ferreira ${ }^{2}$, \\ Daiane Bopp Fuentefria ${ }^{2}$ e Gertrudes Corção ${ }^{1,2}$
}

\begin{abstract}
RESUMO
Acinetobacter spp é um importante patógeno causador de infecções nosocomiais que acomete pacientes imunocomprometidos e capaz de adquirir resistência a antimicrobianos com facilidade. Os esgotos hospitalares são importantes disseminadores de genes de resistência a antimicrobianos para a microbiota ambiental. Neste contexto, 30 cepas de Acinetobacter spp provenientes de efluente de um hospital em Porto Alegre, RS, foram analisados quanto ao perfil de susceptibilidade a $\beta$-lactamases, quinolonas e aminoglicosídeos através de antibiograma e testes de triagem para metalo beta-lactamases e $\beta$-lactamases de espectro estendido. 0 perfil encontrado revela cepas multi-resistentes e que mecanismos de resistência como a produção de $\beta$-lactamases de espectro estendido e bombas de efluxo podem estar presentes nesses isolados.
\end{abstract}

Palavras-chaves: Acinetobacter spp. $\beta$-lactamases de espectro estendido. Efluente hospitalar. Multi-resistência.

\section{ABSTRACT}

Acinetobacter spp is an important pathogen that is responsible for nosocomial infections affecting immunocompromised patients, and it can easily acquire resistance to antimicrobial agents. Hospital sewage is an important means for disseminating genes for resistance to antimicrobial agents, to the microbiota of the environment. Within this context, 30 strains of Acinetobacter spp from the sewage of a hospital in Porto Alegre, Rio Grande do Sul, were analyzed regarding their profile of susceptibility to $\beta$-lactams, quinolones and aminoglycosides, by means of an antibiogram and tests to screen for metallo- $\beta$-lactamases and extended-spectrum $\beta$-lactamases. The profile obtained revealed the presence of multidrug-resistant strains and showed that resistance mechanisms such as the production of extended-spectrum $\beta$-lactamases and efflux pumps may be present in these strains.

Key-words: Acinetobacter spp. Extended-spectrum $\beta$-lactamases. Hospital sewage. Multidrug resistance.

Os esgotos hospitalares não tratados são graves problemas de saúde pública, pois são os grandes responsáveis pelo lançamento de microrganismos patogênicos portadores de genes de resistência a antimicrobianos no ambiente ${ }^{725}$.

Acinetobacter spp é um cocobacilo Gram-negativo, aeróbio restrito, não móvel e um importante patógeno nosocomial oportunista que acomete pacientes imunocomprometidos. É capaz de resistir ao dessecamento, a amplas faixas de temperatura e $\mathrm{pH}^{35}$. As infecções por Acinetobacter spp tornam-se cada vez mais graves com o aparecimento de cepas resistentes aos antimicrobianos. Desde o início de 1970 ,

\footnotetext{
1. Departamento de Microbiologia, Instituto de Ciências Básicas da Saúde, Universidade Federal do Rio Grande do Sul, Porto Alegre, RS. 2. Programa de Pós Graduação em Microbiologia Agrícola e do Ambiente, Universidade Federal do Rio Grande do Sul, Porto Alegre, RS.

Órgãos financiadores: CAPES-PROF e FAPERGS

Endereço para correspondência: $\mathrm{Dr}^{\mathrm{a}}$. Gertrudes Corção. Dept $\mathrm{t}^{0} \mathrm{de}$ Microbiologia/ ICBS/UFRGS. Rua Sarmento Leite 500, Cidade Baixa, 90050-170 Porto Alegre, RS.

Telefax: $55513308-411$

e-mail: corcao@ufrgs.br

Recebido para publicação em 08/09/2008

Aceito em 05/03/2009
}

isolados de Acinetobacter spp (principalmente Acinetobacter baumannii) têm progressivamente acumulado resistência às penicilinas, cefalosporinas, quinolonas e aminoglicosídeos. Conseqüentemente, os carbapenêmicos tornaram-se a terapia de escolha para as infecções graves causadas pelo gênero ${ }^{1}$.

0 principal mecanismo de resistência a $\beta$-lactâmicos é a produção de $\beta$-lactamases cromossomais ou plasmidiais, como a hiper-expressão do gene cefalosporinase cromossomal do tipo $\mathrm{C}(A m p \mathrm{C})^{8}$. A resistência à penicilinas, cefalosporinas e aztreonam é observada principalmente pela expressão de genes de $\beta$-lactamases de espectro estendido (ESBL) e, muitas vezes, responsável pelo fenótipo de multi-resistência ${ }^{3}$.

A resistência aos carbapenêmicos pode ocorrer pela combinação de diferentes mecanismos: diminuição da permeabilidade das membranas externas, alteração na afinidade das proteínas ligadoras de penicilinas (PBPs) e, raramente, pela hiper-expressão de bombas de efluxo. No entanto, as principais formas de resistência aos carbapenêmicos são a expressão de metalo- $\beta$-lactamases (MBLs) ${ }^{28}$, as quais não são hidrolisadas por inibidores comerciais de serino $\beta$-lactamases e, a expressão de oxacilinases (OXA). As enzimas da família OXA conferem 
resistência a ampicilina e cefalotina e são caracterizadas pela alta ação hidrolítica contra oxacilina e cloxacilina e por serem fracamente inibidas por ácido clavulânico ${ }^{322}$.

0 objetivo deste trabalho foi analisar o perfil de suscetibilidade em Acinetobacter spp provenientes de efluente hospitalar da Cidade de Porto Alegre/Rio Grande do Sul (RS), frente a antimicrobianos utilizados na rotina clínica, bem como inferir sobre quais mecanismos de resistência aos $\beta$-lactâmicos estão disseminados neste ambiente, através da leitura interpretativa do antibiograma e dos testes de triagem.

\section{MATERIAL E MÉTODOS}

Isolados bacterianos. Trinta cepas de Acinetobacter spp foram isoladas de amostras de efluente do Hospital São Lucas, em Porto Alegre, RS. Um litro de amostra foi coletado de pontos diferentes de captação do efluente. De cada ponto, $100 \mathrm{~mL}$ foram submetidos a técnica de membrana filtrante (poro de $0,45 \mu \mathrm{m}$ ). A membrana foi imersa em água peptonada e agitada. Alíquotas de $100 \mu \mathrm{L}$ de diluições decimais foram semeadas em ágar MacConkey. Colônias não fermentadoras de lactose foram selecionadas e a confirmação do gênero foi realizada por coloração de Gram, teste de oxidase, crescimento em ágar TSI (Triple Sugar Iron) e amplificação do $16 \mathrm{~S} \mathrm{rDNA}^{6}$.

Teste de susceptibilidade a antimicrobianos. A susceptibilidade das cepas foi testada frente a 13 antimicrobianos: amicacina (30 $\mathrm{g})$, amoxacilina-clavulanato (20/10 $\mathrm{gg})$, aztreonam $(30 \mu \mathrm{g})$, ceftazidima $(30 \mu \mathrm{g})$, cefepime $(30 \mu \mathrm{g})$, cefotaxima $(30 \mu \mathrm{g})$, cefoxitima $(30 \mu \mathrm{g})$, ciprofloxacina $(5 \mu \mathrm{g})$, gentamicina $(10 \mu \mathrm{g})$, imipenem $(10 \mu \mathrm{g})$, meropenem $(10 \mu \mathrm{g})$, piperacilina-tazobactam $(100 / 10 \mu \mathrm{g})$ e ticarcilina-clavulanato (75/10 $\mu$ g), através da metodologia de difusão em ágar e de acordo com as normas do CLSI (Clinical and Laboratory Standards Institute $)^{4}$. Foi realizada leitura interpretativa dos resultados dos antibiogramas ${ }^{12}{ }^{26}$. A cepa Escherichia coli ATCC 25922 foi utilizada como controle de qualidade da metodologia. Foram considerados multi-resistentes os isolados com resistência a, pelo menos, duas classes dos antimicrobianos testados ${ }^{1}$.

Ensaio por aproximação de disco para detecção de metalo- $\beta$-lactamases. A análise fenotípica da produção de metalo- $\beta$-lactamase foi realizada pelo teste de aproximação de discos e utilizou como substratos imipenem e ceftazidima e, como inibidores o ácido etilenodiamino tetra-acético (EDTA) e o ácido 2-mercaptopropiônico ${ }^{2} 1030$.

Ensaio por sobrenadante de cultura para detecção de metalo- $\beta$-lactamases. Para lise celular, um total de $2 \mathrm{~g}$ de crescimento em ágar Müeller Hinton de cada cepa testada foi suspenso em 15mL de tampão Tris-HCl $(50 \mathrm{mM}, \mathrm{pH} 8,0)$ e submetido a 7 ciclos de 15 s de sonicação (20kHz; Ultrasonic Processor, modelo CV33, Cole Parmer). Posteriormente, $1 \mathrm{~mL}$ da suspensão foi submetido a duas centrifugações de 20 minutos a $14.300 \mathrm{~g}$, o sobrenadante foi retirado e utilizado para detecção de MBL. Uma suspensão de células da cepa indicadora (Escherichia coli ATCC 25922), ajustada para a escala 0,5 de Mac Farland $\left(1,5 \mathrm{X} 10^{8} \mathrm{UFC} / \mathrm{mL}\right)$, foi semeada em ágar Müller Hinton. No centro da placa foi depositado um disco de imipenem $(10 \mu \mathrm{g})$. Acima do disco de imipenem, $20 \mu \mathrm{L}$ do sobrenadante foi inoculado em um disco de papel filtro; à esquerda, em outro disco de papel filtro foi colocado $20 \mu \mathrm{L}$ do sobrenadante juntamente com $2 \mu \mathrm{L}$ de $\mathrm{ZnSO}_{4}(1 \mathrm{mM})$; abaixo, $20 \mu \mathrm{L}$ do sobrenadante foi aplicado juntamente com $10 \mu \mathrm{L}$ de EDTA $(0,04 \mathrm{M})$ e a direita do disco de imipenem, foi aplicado tampão Tris-HCl (50mM e pH 8), como controle negativo do teste. A distância entre os discos, de centro a centro, foi de $15 \mathrm{~mm}^{14}$.

Ensaio por disco-difusão dupla para detecção de $\beta$-lactamases de espectro estendido. As cepas foram testadas através da metodologia de disco-difusão dupla. Uma suspensão de 0,5 de Mac Farland $\left(1,5 \times 10^{8} \mathrm{UFC} / \mathrm{mL}\right)$ da cepa testada foi semeada em ágar Müeller Hinton. No centro da placa, colocou-se um disco contendo amoxacilina-clavulanato $(20 / 10 \mu g)$ e outros quatro discos de antimicrobianos marcadores: ceftazidima $(30 \mu \mathrm{g})$,

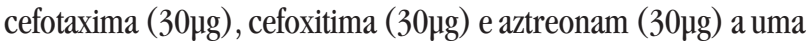
distância de $20 \mathrm{~mm}$ do disco central ${ }^{24}$.

Pesquisa dos genes $\boldsymbol{b l a} \boldsymbol{a}_{\mathrm{VIM}}, \boldsymbol{b l a} \boldsymbol{a}_{\mathrm{IMP}}$ e bla $\boldsymbol{a}_{\mathrm{GES}}$. A pesquisa dos genes $b l a_{\mathrm{VIM}}, b l a_{\mathrm{IMP}}$ e $b l a_{\text {GES }}$ foi realizada pela técnica de reação em cadeia da polimerase (PCR), utilizando como oligonucleotídeos iniciadores: IMP F: 5'-CAT GGT TTG GTG GTT CTT GT- 3' e IMP R: 5'-ATA ATT TGG CGG ACT TTG GC-3', VIM F: 5'- ATG TTC AAA CTT TTG AGT AAG-3' e VIM R: 5'- CTA CTC AAC GAC TGA-3', GES F: 5'-GTT AGA CGG GCG TAC AAA GAT AAT-3' e GES R: 5'- TGT CCG TGC TCA GGA TGA GT-3’’ 0 tamanho esperado das sequiências amplificadas é de $428 \mathrm{pb}$ para $b l a_{\mathrm{IMP}}$, 780pb para $b l a_{\mathrm{VIM}} \mathrm{e}$ 860 pb para $b l a_{\text {GES. }}$. Apenas as cepas com perfil que sugerisse a produção de alguma MBL ou ESBL foram testados.

\section{RESULTADOS}

Das 30 cepas de Acinetobacter spp obtidos a partir de amostras de esgoto hospitalar, $60 \%$ foram considerados multiresistentes. Apenas uma cepa apresentou sensibilidade a todos os antimicrobianos testados. Todos as cepas foram sensíveis a piperacilina-tazobactam. As cepas apresentaram resistência principalmente à amicacina (18/30), aztreonam (10/30), ceftazidima (16/30), cefepime (9/30), ciprofloxacina (17/30), gentamicina (12/30) e ticarcilina-clavulanato $(9 / 30)$. 0 perfil de susceptibilidade das cepas está resumido na Tabela 1. A leitura interpretativa do resultado dos antibiogramas permitiu inferir sobre os possíveis mecanismos de resistência. As cepas deste estudo foram divididos em quatro grupos diferentes, conforme 0 mecanismo de resistência sugerido (Tabela 2).

0 perfil do isolado A11, resistente aos carbapenêmicos, imipenem e meropenem, sugeriu a produção de uma metalo $\beta$-lactamase pela metodologia de aproximação de disco. (Tabela 1). Os resultados do ensaio por sobrenadante de cultura para a produção de MBLs mostraram, a produção de outro tipo de $\beta$-lactamase, que não MBL, em 14 isolados. Foi observada uma distorção de halo em todos os discos, exceto no controle negativo, indicando que as enzimas presentes no 
sobrenadante não são inibidas por EDTA (Figuras 1a e 1b). A triagem para a produção de ESBL pela metodologia discodifusão dupla mostrou que, das 30 cepas testadas, 15 foram positivos para a produção de ESBL (Tabela 1 e Figura 1c) e destes, 5 (A10, A15, A18, A19 e A21) confirmaram a produção de outra $\beta$-lactamase, dado observado também na triagem por sobrenadante de cultura (Tabela 1).

Na reação de PCR, nenhuma das cepas testadas mostrou a presença dos genes $b l a_{\mathrm{IMP}}, b l a_{\mathrm{VIM}}$ e $b l a_{\mathrm{GES}}$, sugerindo a presença de outros mecanismos de resistência.

\section{TABELA 1}

Perfil de susceptibilidade a antimicrobianos e resultado dos testes de triagem fenotípica dos isolados de Acinetobacter spp.

\begin{tabular}{|c|c|c|c|c|c|c|c|c|c|c|c|c|c|c|c|c|}
\hline Isolados & AMI* & ATM & AMC & $\mathrm{CFO}$ & CTX & $\mathrm{CAZ}$ & CPM & CIP & GEN & IPM & MER & PPT & TIC & ESBL $^{\#}$ & $\begin{array}{l}\text { CAZ-2IMPA } \\
\text { IMP-EDTA }^{\#}\end{array}$ & SOBR/BL ${ }^{*}$ \\
\hline A1 & $\mathrm{R}^{\S}$ & $\mathrm{R}$ & $\mathrm{R}$ & $\mathrm{R}$ & $\mathrm{R}$ & $\mathrm{R}$ & $\mathrm{R}$ & $\mathrm{R}$ & $S^{\S}$ & $S$ & $S$ & $S$ & $\mathrm{R}$ & - & - & + \\
\hline A26 & $\mathrm{R}$ & $\mathrm{R}$ & $\mathrm{R}$ & $\mathrm{R}$ & $\mathrm{R}$ & $\mathrm{R}$ & $\mathrm{R}$ & $\mathrm{R}$ & $\mathrm{R}$ & $S$ & $S$ & $S$ & $\mathrm{R}$ & - & - & + \\
\hline A3 & $\mathrm{R}$ & $\mathrm{R}$ & $\mathrm{R}$ & $\mathrm{R}$ & $\mathrm{R}$ & $\mathrm{R}$ & $\mathrm{R}$ & $\mathrm{R}$ & $I^{\S}$ & $S$ & $S$ & $S$ & $\mathrm{R}$ & - & - & - \\
\hline A18 & $\mathrm{R}$ & $\mathrm{R}$ & $\mathrm{R}$ & $\mathrm{R}$ & $\mathrm{R}$ & $\mathrm{R}$ & $\mathrm{R}$ & $\mathrm{R}$ & I & $S$ & S & $S$ & $\mathrm{R}$ & + & - & + \\
\hline A23 & $\mathrm{R}$ & $\mathrm{R}$ & $\mathrm{R}$ & $\mathrm{R}$ & $\mathrm{R}$ & $\mathrm{R}$ & $\mathrm{R}$ & $\mathrm{R}$ & I & $S$ & S & S & $\mathrm{R}$ & - & - & + \\
\hline A81 & $\mathrm{R}$ & I & $\mathrm{R}$ & $\mathrm{R}$ & $\mathrm{R}$ & $\mathrm{R}$ & $\mathrm{R}$ & $\mathrm{R}$ & $\mathrm{R}$ & $S$ & $S$ & $S$ & $\mathrm{R}$ & - & - & + \\
\hline A19 & $\mathrm{R}$ & I & $\mathrm{R}$ & $\mathrm{R}$ & $\mathrm{R}$ & $\mathrm{R}$ & I & $\mathrm{R}$ & $\mathrm{R}$ & $S$ & $\mathrm{~S}$ & $S$ & $\mathrm{R}$ & + & - & + \\
\hline A27/A12 & $\mathrm{R}$ & I & $\mathrm{R}$ & $\mathrm{R}$ & $\mathrm{R}$ & $\mathrm{R}$ & I & $\mathrm{R}$ & $\mathrm{R}$ & $\mathrm{S}$ & S & S & $\mathrm{R}$ & - & - & + \\
\hline A16 & $\mathrm{R}$ & $\mathrm{R}$ & $\mathrm{R}$ & $\mathrm{R}$ & $\mathrm{R}$ & $\mathrm{R}$ & $\mathrm{R}$ & $\mathrm{R}$ & $\mathrm{R}$ & $S$ & S & $S$ & I & - & - & + \\
\hline A11 & $\mathrm{R}$ & I & $\mathrm{S}$ & $\mathrm{R}$ & I & $\mathrm{R}$ & $S$ & $\mathrm{R}$ & $\mathrm{R}$ & $\mathrm{R}$ & $\mathrm{R}$ & $\mathrm{S}$ & $\mathrm{S}$ & + & + & - \\
\hline $\mathrm{A} 20$ & $\mathrm{R}$ & $\mathrm{R}$ & $\mathrm{R}$ & $\mathrm{R}$ & $\mathrm{R}$ & $\mathrm{R}$ & $\mathrm{R}$ & $\mathrm{R}$ & $\mathrm{R}$ & $S$ & S & S & $S$ & - & - & + \\
\hline A5 & $\mathrm{R}$ & I & $\mathrm{R}$ & $\mathrm{R}$ & $\mathrm{R}$ & $\mathrm{R}$ & I & $\mathrm{R}$ & $\mathrm{R}$ & $\mathrm{S}$ & $S$ & $S$ & $\mathrm{~S}$ & - & - & + \\
\hline A15 & $\mathrm{R}$ & I & $\mathrm{R}$ & $\mathrm{R}$ & $\mathrm{R}$ & $\mathrm{R}$ & I & $\mathrm{R}$ & $\mathrm{R}$ & $S$ & S & $S$ & $\mathrm{~S}$ & + & - & + \\
\hline A21 & $\mathrm{R}$ & I & $S$ & $\mathrm{R}$ & $\mathrm{R}$ & $\mathrm{R}$ & $\mathrm{R}$ & $\mathrm{R}$ & $\mathrm{R}$ & $S$ & S & I & $S$ & + & - & + \\
\hline $\mathrm{A} 10$ & $\mathrm{R}$ & I & $\mathrm{R}$ & $\mathrm{R}$ & $\mathrm{R}$ & $\mathrm{R}$ & $\mathrm{S}$ & $\mathrm{R}$ & $\mathrm{R}$ & $\mathrm{S}$ & S & S & $\mathrm{S}$ & + & - & + \\
\hline A32 & $S$ & $\mathrm{R}$ & S & I & S & I & $S$ & $\mathrm{R}$ & $\mathrm{S}$ & $S$ & S & $S$ & $S$ & + & - & - \\
\hline A36 & $\mathrm{R}$ & $\mathrm{R}$ & $\mathrm{R}$ & $\mathrm{R}$ & $\mathrm{R}$ & I & $\mathrm{S}$ & $\mathrm{S}$ & $\mathrm{S}$ & $S$ & $S$ & I & $S$ & - & - & - \\
\hline A63 & $\mathrm{R}$ & $\mathrm{R}$ & $\mathrm{R}$ & $\mathrm{R}$ & $\mathrm{R}$ & $\mathrm{S}$ & $\mathrm{S}$ & $S$ & S & $\mathrm{S}$ & S & $\mathrm{S}$ & $\mathrm{S}$ & - & - & - \\
\hline A17/A31 & $\mathrm{S}$ & I & $\mathrm{R} / \mathrm{S}$ & $\mathrm{R} / \mathrm{S}$ & $\mathrm{I} / \mathrm{S}$ & I & $\mathrm{S}$ & $\mathrm{S}$ & $\mathrm{S}$ & $S$ & $\mathrm{~S}$ & $S$ & $S$ & + & - & - \\
\hline A2/A33 & I & I & $\mathrm{R} / \mathrm{S}$ & $\mathrm{R} / \mathrm{S}$ & I & S & $\mathrm{S}$ & $S$ & S & $S$ & $S$ & $S$ & $S$ & + & - & - \\
\hline A24 & I & S & $\mathrm{S}$ & $\mathrm{S}$ & $\mathrm{S}$ & $\mathrm{S}$ & $\mathrm{S}$ & $S$ & S & $\mathrm{S}$ & S & $S$ & $\mathrm{~S}$ & - & - & - \\
\hline $\mathrm{A} 30$ & I & S & $\mathrm{S}$ & $S$ & I & $\mathrm{S}$ & $\mathrm{S}$ & $\mathrm{S}$ & $\mathrm{S}$ & $S$ & $S$ & $S$ & $S$ & + & - & - \\
\hline A6/A28 & $S$ & I & $S$ & $\mathrm{R} / \mathrm{S}$ & $\mathrm{I} / \mathrm{S}$ & $S$ & $S$ & $S$ & $S$ & $S$ & $S$ & $S$ & $S$ & - & - & - \\
\hline A14/A29 & $S$ & I & $\mathrm{R}$ & $\mathrm{R}$ & I & S & $S$ & $S$ & $S$ & $S$ & $S$ & $S$ & S & + & - & - \\
\hline A4 & $\mathrm{S}$ & $\mathrm{S}$ & $\mathrm{S}$ & I & I & $S$ & $\mathrm{~S}$ & $\mathrm{~S}$ & $S$ & $\mathrm{~S}$ & $S$ & $\mathrm{~S}$ & $S$ & + & - & - \\
\hline
\end{tabular}

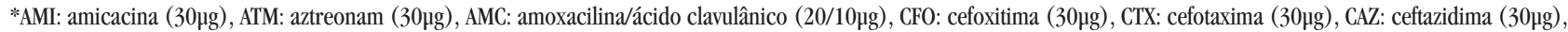

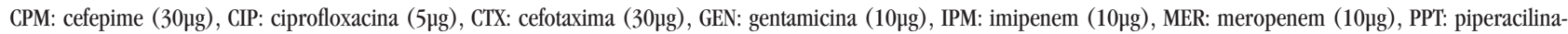

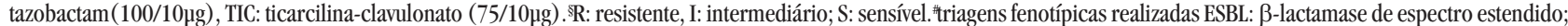
CAZ-2IMPA/ IMP-EDTA: triagem ceftazidima-ácido 2-mercaptopropionico/imipenem EDTA; SOBR/BL: triagem sobrenadante para $\beta$-lactamases.

TABELA 2

Mecanismos de resistência sugeridos pela leitura interpretativa dos isolados de Acinetobacter spp de efluente hospitalar.

\begin{tabular}{lll}
\hline Grupo & \multicolumn{1}{c}{ Isolados } & \multicolumn{1}{c}{ Mecanismos de resistência sugeridos } \\
\hline 1 & $\mathrm{~A} 1, \mathrm{~A} 3, \mathrm{~A} 12, \mathrm{~A} 16, \mathrm{~A} 18, \mathrm{~A} 19, \mathrm{~A} 23, \mathrm{~A} 26, \mathrm{~A} 27, \mathrm{~A} 81$ & TEM-1* ou TEM-2, OXA, sistema de efluxo adeABC \\
2 & $\mathrm{~A} 11$ & Carbapenemase do tipo OXA ou MBL, mutação em gyrA, diminuição dos poros de membrana \\
3 & $\mathrm{~A} 5, \mathrm{~A} 10, \mathrm{~A} 15, \mathrm{~A} 20, \mathrm{~A} 21, \mathrm{~A} 32, \mathrm{~A} 36, \mathrm{~A} 63$ & $\mathrm{AmpC}$, mutação em gyrA em A5, A10, A15, A20, A21 e A32, presença de AAC(6) ou AAC(3) \\
4 & $\mathrm{~A} 2, \mathrm{~A} 4, \mathrm{~A} 6, \mathrm{~A} 14, \mathrm{~A} 17, \mathrm{~A} 24, \mathrm{~A} 28, \mathrm{~A} 29, \mathrm{~A} 30, \mathrm{~A} 31, \mathrm{~A} 33$ & Bombas de efluxo e/ou baixo número de porinas, alteração nas PBPs \\
\hline
\end{tabular}

*AAC(3) AAC(6): enzimas modificantes de aminoglicosídeos do tipo acetiltransferase, adeABC: sistema de bombas de efluxo comumente encontrado em Acinetobacter spp, AmpC: cefalosporinase cromossomal de classe C, gyr A: proteína DNA girase (topoisomerase II) subunidade A, MBL: metalo- $\beta$-lactamase, OXA: $\beta$-lactamase do tipo oxacilinase, PBPs: proteínas ligadoras de penicilinas, TEM 1 e TEM 2: $\beta$-lactamase do tipo temoniera do tipo 1 e 2. 


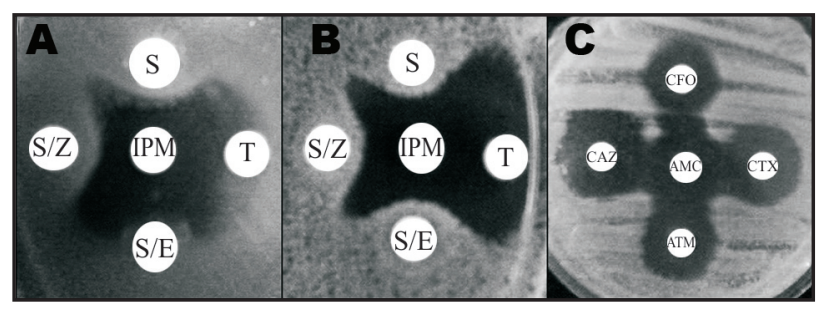

FIGURA 1

Padrões encontrados nas triagens fenotípicas para produção de $\beta$-lactamases em Acinetobacter spp: A: resultado positivo para a produção de metalo- $\beta$-Lactamase no ensaio por sobrenadante de cultura para detecção de metalo- $\beta$-lactamases; B: resultado positivo para a produção de outra $\beta$-lactamase no ensaio do sobrenadante de cultura; C: resultado positivo no ensaio por disco-difusão dupla para detecção de $\beta$-lactamases de espectro estendido, onde se observa uma zona fantasma entre o disco de amoxacilina/ácido clavulânico e cefoxitima. S: sobrenadante, $\mathbf{S} / \mathbf{Z}$ : sobrenadante/ sulfato de zinco, S/E: sobrenadante/EDTA; T: tampão (controle negativo), IPM:

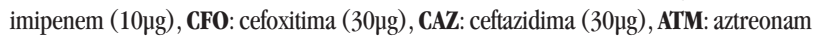

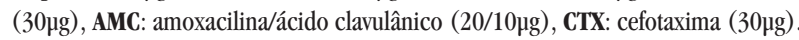

\section{DISCUSSÃO}

A leitura interpretativa do antibiograma ${ }^{12}{ }^{26}$ sugere que 0 perfil de susceptibilidade encontrado nas cepas deste estudo é consequiência, não apenas de mecanismos de resistência isolados, mas da combinação de vários mecanismos, gerando um fenótipo de multi-resistência comumente encontrado em Acinetobacter spp ${ }^{29}$. Por serem cepas isoladas de efluente, tal fenótipo é freqüentemente encontrado, uma vez que tais ambientes propiciam condições adequadas para a troca de informações genéticas que geram tais características $^{725}$. Além disso, este é um ambiente com forte pressão seletiva para cepas bacterianas multiresistentes.

Desta forma, a resistência observada nas cepas do grupo 1 (Tabela 2) está relacionada, provavelmente, com a produção de TEM-1 ou TEM-2, pois essas enzimas, pertencentes ao grupo das ESBLs, conferem resistência a carboxipenicilinas, e ureidopenicilinas, como a ticarcilina e piperacilina ${ }^{26}$. As cepas deste grupo também podem estar relacionados com a presença de uma enzima OXA (não carbapenêmica) ${ }^{3}$, que confere resistência a cefalosporinas, aztreonam, aminoglicosídeos, amoxacilina/ clavulanato, mas não aos carbapenêmicos ${ }^{17}$ e são hidrolisadas por inibidores de serino $\beta$-lactamases comerciais ${ }^{26}$. Em Acinetobacter spp, as ESBLs das famílias TEM, PER, VEB, CTX-M são bastante comuns $^{2027}$. Bombas de efluxo também são freqüentes em Acinetobacter spp, destacando-se o sistema de efluxo AdeABC, que confere resistência aos aminoglicosídeos, cefotaxima, tetraciclina, eritromicina, cloranfenicol, trimetoprim e fluoroquinolonas ${ }^{21}$.

A cepa A11, do grupo 2 (Tabela 2), com resistência a aminoglicosídeos, cefalosporinas, quinolonas e sensibilidade reduzida a aztreonam, também foi resistente aos carbapenêmicos, indicando a presença de carbapenemases do tipo OXA ou MBL, sendo a última pouco freqüente em Acinetobacter $\operatorname{spp}^{11}{ }^{19}$. A não amplificação dos genes $b l a_{\mathrm{VIM}}$ e $b l a_{\mathrm{IMP}}$ nesta cepa, sugere que a mesma não é produtora destas MBLs especificamente. Por outro lado, carbapenemases do tipo OXA são frequientes no gênero Acinetobacter, sendo divididas em quatro subgrupos filogenéticos: tipo OXA-23, tipo OXA-24, tipo OXA-58 e tipo
OXA-51, este último naturalmente encontrado na espécie Acinetobacter baumanni ${ }^{16}$.

No grupo 3 (Tabela 2), o principal mecanismo de resistência inferido é a presença de cefalosporinase cromossomal do tipo $A m p C$, tal enzima confere resistência principalmente a cefalosporinas, como cefoxitima ${ }^{13}{ }^{15}$, além do monobactâmico, aztreonam ${ }^{1}$. 0 perfil encontrado também indica a presença de uma ESBL do tipo GES. Todavia, a ausência de amplificação do gene $b l a_{\text {GES}}$, corrobora a observação de que esta enzima, embora encontrada com frequiência em outras espécies bacterianas no Brasil, é rara no gênero Acinetobacter ${ }^{18}$.

No grupo 4 (Tabela 2), o provável mecanismo que confere resistência à amoxacilina/ácido clavulânico e cefoxitima ou diminuição de sensibilidade à cefotaxima, ceftazidima, aztreonam ou amicacina é a expressão de bombas de efluxo, combinado com o baixo número de porinas e a presença de poros muito pequenos, impedindo a passagem da droga para o interior da membrana $^{2126}$.

Outros mecanismos de resistência a outras classes de antimicrobianos podem estar presentes nas cepas do presente estudo, assim, a resistência a ciprofloxacina é freqüentemente dada por mutações no gene gyrA, que codifica as subunidades A da DNA girase ${ }^{26}$ e a resistência aos aminoglicosídeos, é consequiência provável da presença de enzimas modificantes de aminoglicosídeos do tipo acetiltransferase, AAC (6) e AAC (3), combinadas com a baixa permeabilidade de membrana bacteriana ${ }^{11}$.

A análise das 30 cepas mostra que $50 \%$ foram produtoras de $\beta$-lactamase de espectro estendido. 0 ensaio de disco difusão dupla utilizado neste estudo, padronizado inicialmente para cepas de Klebsiella spp e outras Enterobacteriaceae, como Escherichia coli e Proteus mirabilis, pode gerar falsos positivos e falsos negativos para cepas de Pseudomonas aeruginosa e Acinetobacter $\operatorname{spp}^{23} 24$. Tais erros são resultados inerentes da metodologia do ensaio, baseada na resistência ao clavulanato, já que muitos isolados de Acinetobacter spp mostram resistência intrínseca a esse inibidor $^{12} 23$. Os resultados falso-negativos são decorrentes, entre outros fatores, da presença de $\beta$-lactamases cromossomais do tipo $A m p C$, que inativam as cefalosporinas e, como não sofrem a ação dos inibidores cromossomais, podem mascarar a presença de ESBL $^{1819}$. Das 15 cepas produtoras de ESBL, 10 apresentaram-se negativas para o ensaio por sobrenadante de cultura e isto pode ser resultado da baixa produção das enzimas no sobrenadante, relacionada a diversos fatores, incluindo a formação de estruturas dentro do genoma que interferem na transcrição, resultando na diminuição da expressão genética ${ }^{25}$.

0 número de cepas de Acinetobacter spp multi-resistentes vêm aumentando e a disseminação dessas características para outras bactérias do ambiente é cada vez mais preocupante, uma vez que a microbiota ambiental pode atuar como reservatório de resistência a antimicrobianos ${ }^{6725}$. Os resultados obtidos indicam a presença de Acinetobacter spp com diferentes mecanismos de resistência a $\beta$-lactâmicos no efluente hospitalar estudado, atentando para a importância de programas de tratamento de efluente hospitalar que diminuam a disseminação dessas cepas para o ambiente. 


\section{AGRADECIMENTOS}

Agradecemos à Dr ${ }^{\mathrm{a}}$ Ana Cristina Gales do Laboratório Alerta e Laboratório Especial de Microbiologia Clínica, Divisão de Doenças Infecciosas, Universidade Federal de São Paulo, São Paulo, Brasil, pelo fornecimento das cepas utilizadas como controle positivo para produção de MBL (Metalo- $\beta$-Lactamase) e para a produção de ESBL ( $\beta$-Lactamase de Espetro Estendido) do tipo GES (Guiana-Extended-Spectrum).

\section{REFERÊNCIAS}

1. Abbo A, Navon-Venezia S, Hammer-Muntz 0, Krichali T, Siegman-Igra Y, Carmeli Y. Multidrug-resistant Acinetobacter baumannii. Emerging Infectious Diseases 11:22-29, 2005.

2. Arakawa Y, Shibata N, Shibayama K, Kurokawa H, Yagi T, Fujiwara H, Goto M, Convenient test for screening metallo- $\beta$-lactamase-producing gram-negative bacteria by using thiol compounds. Journal of Clinical Microbiology 38: 40-43, 2000.

3. Brown S, Amyes S. OXA $\beta$-lactamases in Acinetobacter: the story so far. Journal of Antimicrobial Chemotherapy 57:1-3, 2006.

4. Clinical and Laboratory Standards Institute. M100-S17. Performance standards for antimicrobial susceptibility testing; $16^{\text {th }}$ informational supplement. Clinical and Laboratory Standards Institute, Wayne, PA. 2007.

5. Coelho J, Woodford N, Turton J, Livermore DM. Multiresistant Acinetobacter in the UK: how big a threat? Journal of hospital infection 58:167-169, 2004.

6. Ferreira AE, Cunha GR, Fuentefria DB, Corção G. Perfil de suscetibilidade a antimicrobianos em cepas de Acinetobacter spp isoladas de efluente hospitalar em Porto Alegre-RS. Caderno de Farmácia 23:9-14, 2007.

7. Guardabassi L, Petersen A, Olsen JE, Dalsgaard A. Antibiotic resistance in Acinetobacter spp isolated from sewers receiving waste effluent from a hospital and a pharmaceutical plant. Applied and Environmental Microbiology 64:3499-3502, 1998.

8. Héritier C, Poirel L, Lambert T, Nordmann P. Contribution of acquired carbapenem-hydrolyzing oxacillinases to carbapenem resistance in Acinetobacter baumannii. Antimicrobial Agents and Chemotherapy 49:3198-3202, 2005.

9. Jeong SH, Bae IK, Park KO, An YJ, Sohn SG, Jang SJ, Sung KH, Yang KS, Lee K, Young D, Lee SH. Outbreaks of imipenem-resistant Acinetobacter baumannii producing carbapenemases in Korea. Journal of Microbiology 44:423-431, 2006.

10. Lee K, Lim YS, Yong D, Yum JH, Chong Y. Evaluation of the Hodge test and the imipenem-EDTA double-disk synergy test for differentiating metallo- $\beta$-lactamaseproducing isolates of Pseudomonas spp and Acinetobacter spp. Journal of Clinical Microbiology 41:4623-4629, 2003.

11. Livermore DM. Defining an extended-spectrum $\beta$-lactamase. Clinical Microbiology and Infection 14:3-10, 2008.

12. Livermore DM, Winstanley TG, Shannon KP. Interpretative reading: recognizing the unusual and inferring resistance mechanisms from resistance phenotypes. Journal of Antimicrobial Chemotherapy 48:87-102, 2001.
13. Livermore DM, Woodford $\mathrm{N}$. The $\beta$-lactamase threat in Enterobacteriaceae, Pseudomonas and Acinetobacter. TRENDS in Microbiology 14:413-420, 2006.

14. Marchiaro P, Mussi MA, Ballerini V, Pasteran F, Viale AM, Vila AJ, Limansky AS. Sensitive EDTA-based microbiological assays for detection of metallo- $\beta$ lactamases in nonfermentative gram-negative bacteria. Journal of Clinical Microbiology 43:5648-5652, 2005.

15. Mendes RE, Castanheira M, Pignatari ACC, Gales AC. Metalo- $\beta$-lactamases. Jornal Brasileiro de Patologia e Medicina Laboratorial 42:103-113, 2006.

16. Mezzatesta ML, Trovato G, Gona F, Nicolosi VM, Nicolosi D, Carattoli A, Fadda G, Nicoletti G, Stefani S. In vitro activity of tigecycline and comparators against carbapenem-susceptible and resistant Acinetobacter baumannii clinical isolates in Italy. Annals of Clinical Microbiology and Antimicrobials 7: 1-7, 2008.

17. Naas T, Poirel L, Nordmann P. Minor extended-spectrum $\beta$-lactamases. Clinical Microbiology and Infection 14:42-52, 2008.

18. Picão RC, Gales AC. $\beta$-lactamases de espectro ampliado (ESBL) Pseudomonas aeruginosa: pesadelo ou só imaginação? Prática Hospitalar 49:79-84, 2007.

19. Pino IC, Domínguez YM, González RG, Bello TH, Sepúlveda AM, Mella MS, Zemelman MC, Zemelman ZR. Extended spectrum $\beta$-lactamases (ESBL) production in Acinetobacter baumannii strains isolated from Chilean hospitals belonging to VIII Region. Revista Chilena de Infectología 24:137-141, 2007.

20. Poirel L, Naas T, Nordmann P. Genetic support of extended-spectrum $\beta$-lactamases. Clinical Microbiology and Infection 14: 75-81, 2008.

21. Poole K. Efflux-mediated multiresistance in Gram-negative bacteria. Clinical Microbiology and Infection 10:12-26, 2004.

22. Pournaras S, Markogiannakis A, Ikonomidis A, Kondyli L, Bethimouti K, Maniatis AN, Legakis NJ, Tsakris A Outbreak of multiple clones of imipenem-resistant Acinetobacter baumannii isolates expressing OXA-58 carbapenemase in an intensive care unit. Journal of Antimicrobial Chemotherapy 57:557-561, 2006.

23. Sampaio JLM. Consenso em detecção de resistência bacteriana aos antimicrobianos em bacilos Gram-negativos. Revista da Sociedade Brasileira de Microbiologia 3:18-27, 2008.

24. Shah AA, Hasan F, Ahmed S, Hameed A. Prevalence of extended-spectrum $\beta$-lactamases in nosocomial and outpatients (ambulatory). Pakistan Journal of Medical Sciences 19:187-219, 2003.

25. Tennstedt T, Szczepanowski R, Braun S, Pühler A, Schlüter A. Occurrence of integron-associated resistance gene cassettes located on antibiotic resistance plasmids isolated from a wastewater treatment plant. FEMS Microbiology Ecology 45:239-252, 2003

26. Vila J, Marco F. Interpretative reading of the non-fermenting gram-negative bacilli antibiogram. Enfermedades Infecciosas y Microbiología Clínica 20:304-312, 2002.

27. Villegas MV, Kattan JN, Quinteros MG, Casellas JM. Prevalence of extendedspectrum $\beta$-lactamases in South America. Clinical Microbiology and Infection 14:154-158, 2008.

28. Walsh TR, Toleman MA, Poirel L, Nordmann P. Metallo- $\beta$-lactamases: the quiet before the storm. Clinical Microbiology Reviews 18:306-325, 2005.

29. Yin XL, Hou TW, Xu SB, Ma CQ, Yao ZY, Li W, Wei L. Detection of Drug ResistanceAssociated Genes of Multidrug-Resistant Acinetobacter baumannii. Microbial Drug Resistance 14:145-150, 2008.

30. Yong D, Lee K, Yum JH, Shin HB, Rossolini, GM, Chong, Y. Imipenem-EDTA disk method for differentiation of metallo- $\beta$-lactamase-producing clinical isolates of Pseudomonas spp and Acinetobacter spp. Journal of Clinical Microbiology 40:3798-3801, 2002 